\title{
Medication adherence in patients with psychotic disorders: an observational survey involving patients before they switch to long-acting injectable risperidone
}

\author{
This article was published in the following Dove Press journal: \\ Patient Preference and Adherence \\ 16 September 2015 \\ Number of times this article has been viewed
}

\author{
Franck Jean Baylél \\ Arnaud Tessier ${ }^{2,3}$ \\ Sophie Bouju ${ }^{4}$ \\ David Misdrahi ${ }^{2,3}$ \\ 'Sainte-Anne Hospital (SHU), Paris \\ V-Descartes University, Paris, ${ }^{2}$ Hôpital \\ Charles Perrens, Pôle de Psychiatrie \\ Adulte, ${ }^{3}$ CNRS UMR 5287-INCIA, \\ Bordeaux University, Bordeaux, \\ ${ }_{4}^{4}$ Janssen-Cilag France, Issy Les \\ Moulineaux, Paris, France
}

\begin{abstract}
Background: Maintaining antipsychotic therapy in psychosis is important in preventing relapse. Long-acting depot preparations can prevent covert non-adherence and thus potentially contribute to better patient outcomes. In this observational survey the main objective is to evaluate medication adherence and its determinants for oral treatment in a large sample of patients with psychosis.
\end{abstract}

Methods: In this cross-sectional survey medication adherence for oral treatment was assessed by patients using the patient-rated Medication Adherence Questionnaire (MAQ). Data were collected by physicians on patients with a recent acute psychotic episode before switching to long-acting injectable risperidone. Other evaluations included disease severity (Clinical Global Impression - Severity), patients' insight (Positive and Negative Syndrome Scale item G12), treatment acceptance (clinician-rated Compliance Rating Scale), and therapeutic alliance (patient-rated 4-Point ordinal Alliance Scale).

Results: A total of 399 psychiatrists enrolled 1,887 patients (mean age $36.8 \pm 11.9$ years; $61.6 \%$ had schizophrenia). Adherence to oral medication was "low" in $53.2 \%$ of patients, "medium" in $29.5 \%$, and "high" in $17.3 \%$. Of patients with psychiatrist-rated active acceptance of treatment, $70 \%$ had "medium" or "high" MAQ scores $(P<0.0001)$. Medication adherence was significantly associated with therapeutic alliance (4-Point ordinal Alliance Scale score; $P<0.0001$ ). Patient age was significantly associated with adherence: mean age increased with greater adherence (35.6, 36.7, and 38.6 years for patients with "low", "medium", and "high" levels of adherence, respectively; $P=0.0007)$, while age $<40$ years was associated with "low" MAQ classification $(P=0.0003)$. Poor adherence was also associated with a diagnosis of schizophrenia $(P=0.0083)$, more severe disease (Clinical Global Impression - Severity $\geq 4 ; P<0.0001$ ), and lower insight (Positive and Negative Syndrome Scale-G12 $\geq 4 ; P<0.0001$ ).

Conclusion: Self-reported adherence was low in most patients, with a strong positive association between self-reported adherence and psychiatrists' assessment of treatment acceptance. Understanding factors associated with poor medication adherence may help physicians to better manage their patients, thereby improving outcomes.

Keywords: schizophrenia, long-acting antipsychotic, medication adherence, therapeutic alliance

\section{Background}

Schizophrenia is a chronic, severe, and disabling disorder. Although most patients recover symptomatically from their first episode, the majority will experience subsequent psychotic relapses. ${ }^{1}$ Disease progression typically involves multiple cycles of exacerbation 
and remission, and patients may have incomplete recovery from psychotic relapses, thereby accumulating morbidity; ${ }^{1}$ progressive functional deterioration can thus occur.

Long-term continuous treatment is required to achieve and sustain remission. ${ }^{2}$ Early therapeutic intervention and long-term maintenance of medication are predictive of better outcomes and fewer relapses. ${ }^{1,3}$ Furthermore, the early phase of psychosis may present important opportunities for secondary prevention - critical psychosocial influences, including psychological reactions to psychiatric services, develop during this period. ${ }^{4}$

One of the main barriers to successful management of schizophrenia, which is relatively common among patients with this condition, is poor adherence to medication. ${ }^{2,5-8}$ This is a major (yet potentially modifiable) risk factor for relapse, hospitalization, and even suicide attempts. 2,6,7,9,10

Medication adherence is a complex issue affected by many factors, and adherence problems are multi-determined..$^{8,11}$ Prediction of risk factors for these problems may help to facilitate strategies for improved adherence. ${ }^{9}$ Poor insight/ lack of illness awareness has been reported as a key factor for non-/partial adherence or negative attitudes toward antipsychotic medication..$^{5,8,12-14}$ In addition to lack of insight, the clinician's style of communication and therapeutic alliance (patient-clinician relationship) are important.,15-18 Indeed, weak therapeutic alliance and low patient insight have been shown to be significantly associated with poor adherence in patients with schizophrenia or schizoaffective disorder. ${ }^{7}$ Consequently, to improve adherence and outcomes, psychoeducational programs aiming to improve therapeutic alliance and insight should be implemented. ${ }^{7}$

Because maintaining antipsychotic therapy is also a key element in preventing relapse, long-acting depot preparations offer a useful therapeutic option; ${ }^{19,20}$ these can prevent covert non-adherence and thus potentially contribute to better patient outcomes. ${ }^{21-23}$ In this cross-sectional survey the main objective was to evaluate medication adherence and its determinants for oral treatment in a large sample of patients with psychosis after an acute episode and just before switching to long-acting injectable risperidone. Understanding factors associated with poor medication adherence may help physicians to better manage their patients, thereby improving outcomes.

\section{Methods}

\section{Study design and patients}

This study was an observational survey, being performed under "real-life" conditions in accordance with applicable guidelines.
The study received approval from the local human research committee. All patients provided written informed consent before data collection. While the initial data collection took place between April 2007 and June 2008, the full statistical analysis described in the current manuscript was competed in October 2012.

From an initial pool of 5,500 psychiatrists working in public or private clinics throughout France, a representative sub-sample of 1,500 individuals was selected, weighted by geographic region. The psychiatrists routinely treated both hospitalized individuals and outpatients for psychotic disorders. By randomization, a total of 500 psychiatrists from the diverse regions were then asked to systematically collect observations for at least the first four patients assessed who met the inclusion criteria. Of these 500 psychiatrists, 399 actively participated in the survey and included 1,887 patients, representing a mean of 4.7 patients per psychiatrist. To be included in this survey, patients were required to be hospitalized or community-dwelling individuals with psychosis (International Classification of Diseases [ICD]-10), diagnosed with a recent acute psychotic episode that had been stabilized using oral risperidone before a subsequent switch to long-acting injectable risperidone was initiated. Additional patient inclusion criteria included age $\geq 18$ years and fluency in the French language with the ability to understand the study protocol. Exclusion criteria included traumatic head injury, any past or present major medical or neurological illness, and mental retardation.

The treating psychiatrist, using a standardized semistructured questionnaire, collected baseline sociodemographic and clinical information. Disease severity, patients' levels of insight into their condition, and treatment acceptance were evaluated by the treating psychiatrist as described below, together with a self-report patient questionnaire which assessed medication adherence and therapeutic alliance. All the participants were evaluated after the acute phase in a single interview of approximately 45 minutes.

\section{Outcome measures}

\section{Disease severity}

Disease severity was assessed by the psychiatrist using the "severity of illness" section of the Clinical Global Impression Scale (CGI-S). ${ }^{24}$ The CGI-S scale is a well-known and relatively straightforward single-item instrument used to assess the overall (global) severity of illness as a graded measure of increasing psychopathology from 1 to 7 . The original description of the CGI-S provided the progressive seven-point range of scores but did not offer scoring anchors to standardize scoring between raters. Recently, published 
scoring guidelines have improved both inter-rater reliability and the precision of CGI scoring $(r=0.79) .{ }^{25}$ The CGI-S scale offers a readily understood, practical measurement tool that can be easily administered by a clinician in a busy clinical practice setting.

\section{Insight}

The Positive and Negative Syndrome Scale (PANSS) is a 30 -item rating scale for use by clinically trained research staff. ${ }^{26}$ In this study, insight was evaluated using the G12 item of the PANSS ("lack of judgment and insight"). This is rated from 1 to 7 , representing increasing levels of psychopathology: $1=$ absent; $2=$ minimal; $3=$ mild; $4=$ moder ate; $5=$ moderately severe; $6=$ severe $7=$ extremely severe. PANSS-G12 scores reflect global awareness of symptoms, treatment need, and consequences of illness, with higher scores indicating lower insight.

\section{Medication adherence}

Medication adherence was evaluated using the Medication Adherence Questionnaire (MAQ), which considers the patient's medication-taking behavior over the previous week. ${ }^{27}$ For the purpose of the current study, adherence for outpatients was based on the week before interview whereas inpatients were asked to respond by referring to adherence behavior before hospitalization. Completed by the patient, this is a short, simple tool comprising four items:

1) Do you ever forget to take your medication?

2) Are you sometimes careless about taking medication?

3) When you feel better, do you sometimes stop taking medication?

4) Sometimes when you feel worse, do you stop taking your medication?

Each question is answered "Yes" or "No", and each "Yes" answer receives a score of 1 , with the total scoring ranging from 0 to 4 . The total score permits classification of level of adherence into three discrete categories: "low" (three or four "Yes" answers), "medium" (one or two "Yes" answers), or "high" (no "Yes" answers).

\section{Treatment acceptance}

Each patient's level of treatment acceptance was assessed by his/her treating psychiatrist using a clinician-rated 7-point ordinal scale (Compliance Rating Scale [CRS]) as described by Kemp et al. ${ }^{28}$ Patients who completely refused treatment were assigned a score of 1 ; patients who partially refused treatment were assigned a score of 2 ; reluctant, occasional or passive acceptance of treatment were scored as 3, 4 or 5, respectively; moderate or active participation in treatment acceptance received scores of 6 and 7, respectively. With this outcome measure, a score $\geq 5$ was regarded as indicative of adherence.

\section{Therapeutic alliance}

The 4-Point ordinal Alliance Scale (4PAS) was used to assess therapeutic alliance ${ }^{17}$ it includes elements relating to a patient's relationship with his/her clinician on a day-today basis and to medication. Completed by the patient, the 4PAS is amenable for use in clinical psychiatric practice. It comprises an 11-item questionnaire, which is scored using a 4-point Likert scale ranging from 1 ("strongly disagree") to 4 ("strongly agree"). The total score ranges from 11 to 44, with a higher 4PAS score indicating better therapeutic alliance.

\section{Statistical methods}

Results were recorded anonymously for subsequent data processing, which was conducted using SAS software, version 8.2 (SAS Institute Inc., Cary, NC, USA). ${ }^{29}$

Sociodemographic and clinical variables are presented descriptively. Quantitative parameters are described with means, medians, standard deviations (SDs), and ranges. Qualitative parameters are presented in terms of frequencies and percentages.

The Kolmogorov-Smirnov one-sample test was used to verify the distribution of the data. Differences between groups were investigated with tests applicable to the variables under consideration; these included the Student's $t$-test and MannWhitney-Wilcoxon tests for normally and non-normally distributed continuous variables, respectively, and the $\chi^{2}$ or Kruskal-Wallis test, as appropriate, for categorical variables, as shown in the "Results" section. Multivariate logistic regression analyses were performed on clinical variables with $P$-values $<0.05$ in the univariate analysis and quantified with odds ratios and their $95 \%$ confidence intervals.

\section{Results \\ Sociodemographic and clinical characteristics of the study participants}

In total, 399 psychiatrists participated in this survey (mean \pm SD age of $44.7 \pm 8.2$ years; median 45 years, range 28-64 years), the majority of whom $(57.9 \%)$ were male.

The survey also involved 1,887 patients (mean \pm SD age of 36.8 \pm 11.9 years; median 35 years, range $18-90$ years), and the majority of these $(64.3 \%)$ were also male (Table 1$)$. Most of the patients had been diagnosed with schizophrenia (61.6\%), although schizoaffective disorder (19.3\%) and other psychotic disorders (19.1\%) had been identified. More than half the patients $(57.8 \%)$ were hospitalized, $42.2 \%$ were 
Table I Demographic characteristics of the patients

\begin{tabular}{ll}
\hline Characteristic & \\
\hline Age in years, mean \pm SD & $36.8 \pm 1$ I.9* \\
Sex, $\mathrm{n}(\%)$ patients & \\
$\quad$ Male & $\mathrm{I}, 210(64.3)$ \\
$\quad$ Female & $673(35.7)$ \\
Marital status, $\mathrm{n}(\%)$ patients & \\
$\quad$ Single & $\mathrm{I}, 279(68.0)$ \\
$\quad$ Married or in partnership & $335(17.8)$ \\
$\quad$ Divorced, separated, or widowed & $266(14.1)$ \\
Living arrangements, $\mathrm{n}(\%)$ patients & \\
$\quad$ Living independently & $\mathrm{I}, 014(54.5)$ \\
$\quad$ Institutionalized & $729(39.2)$ \\
$\quad$ No fixed residence & $61(3.3)$ \\
$\quad$ Other & $55(3.0)$ \\
Occupational status, $\mathrm{n}(\%)$ patients & \\
$\quad$ Unemployed & $\mathrm{I}, 440(76.9)$ \\
Employed & $433(23.1)$ \\
\hline
\end{tabular}

Note: $*_{n}=1,872$.

Abbreviation: SD, standard deviation.

outpatients, and $38.8 \%$ of the sample had a monthly consultation with a psychiatrist.

The two main reasons to start the long-acting injectable risperidone were related to non-compliance with oral antipsychotic treatment $(92.4 \%)$ and intention to improve efficacy (86.4\%). Maintenance of a good therapeutic alliance $(70.3 \%)$ and treatment tolerability $(54.6 \%)$ were also often cited. According to the CGI-S, $67.7 \%$ of the patients were "moderately ill" or "markedly ill", and $21.1 \%$ were "severely ill" or "among the most extremely ill".

Insight (PANSS-G12 scores) was considered "normal" in $36.6 \%$ of patients, "moderate" in $34.8 \%$, and "low" in $28.6 \%$.

\section{Medication adherence (MAQ data)}

Medication adherence was evaluated in a total of 1,610 patients $(85.3 \%)$ who completed the MAQ. Adherence to oral medication, before initiating long-acting injectable risperidone, was "low" in $53.2 \%$ of patients, "medium" in $29.5 \%$, and "high" in $17.3 \%$.

The MAQ results also showed that $64.0 \%$ of patients occasionally forgot to take their medication (Item 1), 63.6\% neglected the correct time for taking the medication (Item 2), and $60.7 \%$ sometimes interrupted their medication when feeling better (Item 3).

\section{Medication adherence (MAQ) and treatment acceptance (CRS)}

As assessed by the treating psychiatrist, $41.6 \%$ of patients demonstrated some degree of reticence or reluctance toward receiving treatment, while $58.4 \%$ had a passive, moderately active, or active acceptance of treatment (CRS data).

A strong positive association was apparent between medication adherence as reported by patients ("medium" or "high" MAQ scores) and treatment acceptance as assessed by the psychiatrists $\left(P<0.0001, \chi^{2}\right.$ test $): 70 \%$ of patients with active acceptance of treatment had "medium" or "high" MAQ scores.

\section{Medication adherence (MAQ) and therapeutic alliance (4PAS)}

Evaluating therapeutic alliance using the 4PAS, the mean score was $35.8 \pm 5.9$ (median 36 , range $11-44$ ). The internal consistency of this scale was excellent (Cronbach's $\alpha=0.92$ ).

Medication adherence, "low", "medium", or "high" as evaluated in terms of the MAQ, was significantly associated with therapeutic alliance, as measured by 4PAS score $(P<0.0001)$ (Figure 1).

\section{Demographic factors associated with medication adherence}

Considering levels of adherence in terms of "low", "medium", and "high" MAQ classes, a statistically significant association was apparent with regard to patient age, with the mean age increasing with higher levels of adherence (35.6, 36.7, and 38.6 years for patients with "low", "medium", and "high" levels of adherence, respectively; $P=0.0007)$. No relationship was found with regard to patient sex, marital status and living arrangements or occupation. Neither the age nor the sex of the

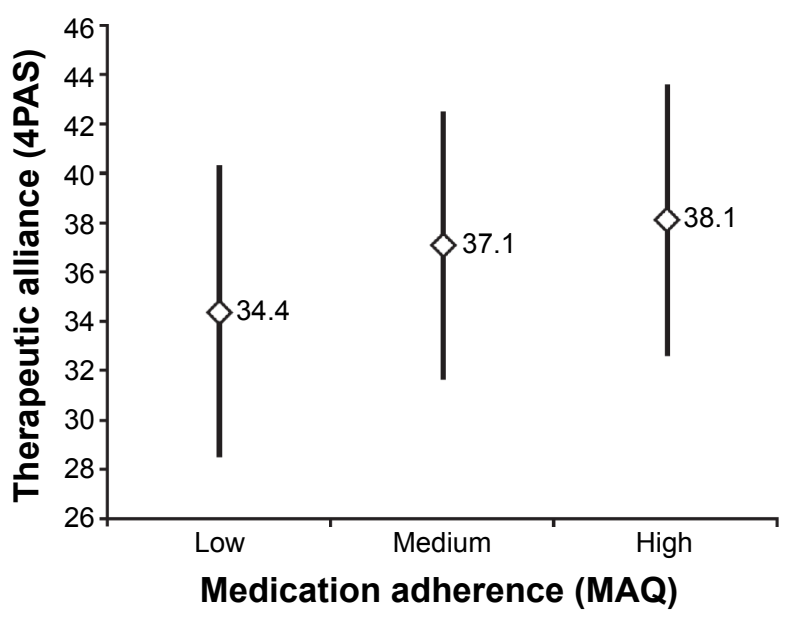

Figure I Medication adherence (MAQ) according to therapeutic alliance (4PAS). Notes: Medication adherence (MAQ data) was significantly associated with therapeutic alliance $(P<0.000 \mathrm{I})$. Data are presented as mean \pm standard deviation. Abbreviations: MAQ, Medication Adherence Questionnaire; 4PAS, 4-Point ordinal Alliance Scale. 
treating psychiatrists influenced patients' adherence levels as measured in terms of these three MAQ classes.

\section{Other clinical variables}

Poor medication adherence was strongly associated with a diagnosis of schizophrenia $\left(P=0.0083, \chi^{2}\right.$ test $)$, more severe disease (CGI-S $\geq 4 ; P<0.0001, \chi^{2}$ test), and lower insight (PANSS-G12 $\geq 4 ; P<0.0001, \chi^{2}$ test). Multivariate analyses confirmed that patients with the best adherence ("medium + high" MAQ) were significantly older $(P<0.001)$ whereas bad compliers ("low" MAQ) had a more severe disease $(P<0.0001)$, and poorer insight $(P<0.0001)$ (Table 2).

\section{Factors associated with low therapeutic alliance (4PAS)}

Therapeutic alliance was lower in "severely ill" or "among the most extremely ill" patients $(P<0.001$, Kruskal-Wallis test), and in those with a lack of insight $(P<0.001$, KruskalWallis test) (Figure 2).

\section{Discussion}

This observational study evaluated 1,887 patients with psychotic disorders treated by 399 psychiatrists. Patients were required to be switching from oral treatment to long-acting injectable risperidone, a switch that for around $90 \%$ of the treating psychiatrists had been prompted by their patients' non-adherence to oral therapy and an intention to improve efficacy. Long-acting, injectable preparations have been developed in efforts to enhance adherence and to stabilize drug plasma levels. ${ }^{30}$

The perceptions of psychiatrists switching their patients to long-acting injectable risperidone were borne out by additional study data: self-reported adherence to oral risperidone was low in the majority of patients (53.0\%). Furthermore, a strong positive association was apparent between patients' self-reported adherence (MAQ data) and their psychiatrists' assessment of treatment acceptance (CRS data). However, these data were obtained from a selective sample population in which clinicians had frequently decided to switch treatment to long-acting injectable risperidone to prevent non-adherence to oral medication; they contrast other published results whereby agreement between patients' and physicians' evaluations of adherence to antipsychotic medication has been low. ${ }^{31}$ Treatment non-adherence is often underestimated by psychiatrists. ${ }^{32}$ Kikkert et al ${ }^{33}$ used concept mapping to explore the patients', professionals', and carers' views of medication adherence in schizophrenia, identifying five clinically relevant factors that affected medication adherence (efficacy of medication, external factors, insight,

Table 2 Characteristics statistically significantly associated with poor medication adherence (“low” MAQ classification)

\begin{tabular}{|c|c|c|c|c|c|c|c|}
\hline \multirow[t]{3}{*}{ Variables } & \multicolumn{3}{|c|}{ Univariate model } & \multicolumn{4}{|c|}{ Multivariate model } \\
\hline & \multirow{2}{*}{$\frac{\text { Low MAQ }}{\mathrm{n}(\%)}$} & \multirow{2}{*}{$\frac{\text { Medium + high MAQ }}{\text { n (\%) }}$} & \multirow[t]{2}{*}{$P$-value } & \multirow[t]{2}{*}{ aOR } & \multicolumn{2}{|l|}{$95 \% \mathrm{Cl}$} & \multirow[t]{2}{*}{ P-value } \\
\hline & & & & & & & \\
\hline \multicolumn{8}{|l|}{ Sex } \\
\hline Male & $547(52.6)$ & $492(47.4)$ & 0.5654 & & & & \\
\hline Female & $307(54.1)$ & $260(45.9)$ & & & & & \\
\hline \multicolumn{8}{|l|}{ Age } \\
\hline$<40$ years & $587(56.5)$ & $452(43.5)$ & 0.0003 & 1.566 & 1.313 & 1.869 & 0.0002 \\
\hline$\geq 40$ years & $264(47.1)$ & $296(52.9)$ & & & & & \\
\hline No & 761 (54.7) & $629(45.3)$ & & & & & \\
\hline \multicolumn{8}{|l|}{ Disease severity } \\
\hline CGI-S $\geq 4$ & $785(55.8)$ & $623(44.2)$ & $<0.0001$ & 1.986 & 1.518 & 2.598 & $<0.000$ I \\
\hline CGI-S $<4$ & $69(35.8)$ & $124(64.2)$ & & & & & \\
\hline \multicolumn{8}{|l|}{ Psychotic disorder } \\
\hline Schizophrenia & $555(56.3)$ & 431 (43.7) & 0.0083 & & & & \\
\hline Schizoaffective disorder & $165(52.2)$ & I5I (47.8) & & & & & \\
\hline Persistent delusions & $71(45.8)$ & $84(54.2)$ & & & & & \\
\hline Acute psychotic episode & $47(44.8)$ & $58(55.2)$ & & & & & \\
\hline Other & $15(38.5)$ & $24(61.5)$ & & & & & \\
\hline \multicolumn{8}{|l|}{ Insight } \\
\hline PANSS-GI $2 \geq 4$ & $572(57.8)$ & $417(42.2)$ & $<0.000$ I & 1.459 & 1.225 & 1.738 & 0.0003 \\
\hline PANSS-GI $2<4$ & $279(46.2)$ & $325(53.8)$ & & & & & \\
\hline
\end{tabular}

Abbreviations: MAQ, Medication Adherence Questionnaire; CGI-S, Clinical Global Impression Scale-Severity; PANSS-GI2, Positive and Negative Syndrome Scale (lack of judgment and insight); aOR, adjusted odds ratio; $\mathrm{Cl}$, confidence interval. 


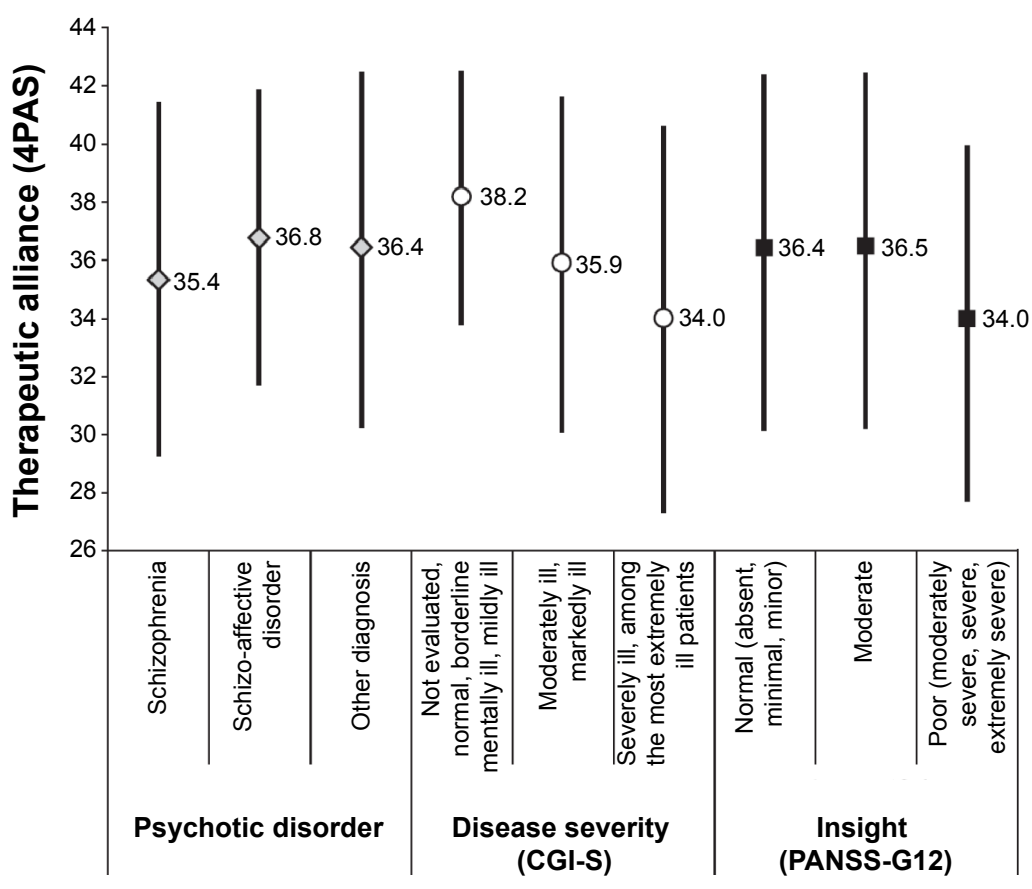

Figure 2 Therapeutic alliance according to psychotic disorder, disease severity (CGI-S), and insight (PANSS-GI2).

Notes: Therapeutic alliance was lower in "severely ill" or "among the most extremely ill" patients $(P<0.00 \mathrm{I})$, and in patients with poor insight $(P<0.00 \mathrm{I})$. Data are presented as mean \pm standard deviation.

Abbreviations: 4PAS, 4-Point ordinal Alliance Scale; CGI-S, Clinical Global Impression Scale-Severity; PANSS-G I2, Positive and Negative Syndrome Scale (lack of judgment and insight).

side effects, and medication attitudes), but the three groups did not have a shared understanding of which factors were important. In light of such information it is important to realize that physicians can positively influence adherence by fostering therapeutic alliance. This is considered in more detail below.

Adherence can be assessed by three methods: auto or hetero-questionnaires, pill counts $\left(\mathrm{MEMS}^{\circledR}\right)$, and biological assay of the active substance or its metabolites. ${ }^{34}$ Selfreported measures are generally considered as the best cost-effective and time-efficient way to assess medication adherence, while it has been reported to sometimes overestimate adherence. ${ }^{8}$ With the pill count, a direct measure of adherence may be obtained but the tablet removal from container cannot be considered as equivalent of a drug intake. Plasma levels are limited by pharmacokinetic biases, and by a phenomenon known as "white-coat adherence", in which patients improve their medication-taking behavior in the 5 days before and after an appointment with the health care provider. ${ }^{34}$ Moreover, this method could be costly and difficult to use in current practice or in large cohorts studied in clinical settings. Finally therapeutic adherence is a dynamic process with no measurement gold standard. ${ }^{8}$ The fact that the sample was comprised of both inpatient and outpatient participants renders the assessment of adherence by self-report more complex as hospitalized patients are more closely monitored than outpatients. However, while no gold standard exists for this issue, the MAQ self-report questionnaire was selected as the most cost-effective instrument for large samples, although it can overestimate adherence or increase recall biases. To reduce this risk, adherence behavior for outpatients was assessed over the week prior to interview whereas inpatients were asked to describe adherence behavior just before hospitalization.

\section{Consideration of factors affecting medication adherence}

Evaluating factors affecting adherence for the treatment of an acute psychotic episode in the current patient population has shown a significant association with therapeutic alliance. Indeed, research has repeatedly demonstrated the importance of the therapeutic alliance in achieving adherence to medication. ${ }^{7,17,35,36}$ Moreover, Wittorf et a ${ }^{37}$ found therapeutic alliance in the treatment of schizophrenia spectrum disorders to be affected by both insight and symptoms, with greater insight and fewer positive and negative symptoms being significant predictors of a higher alliance. In our survey, patient-reported therapeutic alliance was lower in "severely ill" or "among the most extremely ill" patients, and for those with a lack of insight (which, as described below, is 
also in line with the observed associations with medication adherence). Interestingly, Wittorf et $\mathrm{al}^{37}$ also reported that patients' and therapists' perceptions of the alliance did not appear to demonstrate much convergence - patients' ratings were significantly higher.

Psychiatrists can influence therapeutic alliance, which may be expected to improve medication adherence, and hence outcomes, in patients with schizophrenia. In relation to this, it should be noted that regular monitoring of patients also helps to foster a positive therapeutic relationship, contributing to therapeutic alliance, and long-acting injectable preparations may help in this regard (beyond considerations relating directly to adherence and efficacy). ${ }^{20}$ Indeed, Rossi et $\mathrm{al}^{38}$ consider that the use of long-acting injectable medication may help to establish therapeutic alliance via improved adherence.

As mentioned above, more than half the patients in this study were estimated to have poor adherence to oral medication (MAQ data). The reasons for this are likely to be complex and numerous. As reported elsewhere, patient age appears to be a factor, ${ }^{39,40}$ with younger patients being less adherent, as we have found. We also found patients' poor insight into their disorder to be associated with poor adherence, which concurs with other published results..$^{35,41-43}$ Indeed, it has been reported that lack of insight is linked to greater severity of psychopathology, ${ }^{44,45}$ as well as to poor medication adherence, which in turn is associated with poor clinical outcomes, such as frequent relapses and hospitalizations. ${ }^{2,5,8} \mathrm{~A}$ recent naturalistic, longitudinal study of patients with first-episode psychosis suggested that, as better insight improved medication adherence, with consequent benefits on clinical course and functional outcome, insight could be a specific target of early intervention. ${ }^{46}$

Our data have also shown a significant association between increasing disease severity and decreasing adherence to medication, although the cross-sectional nature of our study design does not allow any definitive conclusions on causality to be drawn from these results. Others have also shown disease severity to impact on patients' adherence to their antipsychotic medication, ${ }^{9,47}$ but the degree of correlation between these variables remains to be clearly established. Indirect evidence relating to this is provided by studies in which patients demonstrated better adherence following improvement in psychopathological symptoms. ${ }^{17,48}$ However, Staring et $\mathrm{al}^{49}$ have reported that while adherence to antipsychotic medication may reduce symptoms in patients with schizophrenia, it may also increase side effects, thereby having opposing effects on perceived quality of life.
Yang et $\mathrm{al}^{50}$ have recently concluded that both disease severity and attitudes toward medication are related to adherence, and that further studies are needed.

It is appropriate to consider the limitations of the study and the external validity of the results. No data were recorded about the number of patients (and the reason) who refused to participate. The variability of the sample with in- and outpatient condition, undermine the adherence assessment with self-report. However, with no gold standard to date the reliable self-report questionnaire MAQ was retained as the best cost-effective tool even it can overestimate adherence or generate a recall bias. To reduce this risk of variability, adherence for outpatients was based on the week before interview whereas inpatients were asked to respond by referring to adherence behavior before hospitalization. Definition of acute episode was based on psychiatrists' positive answers to declarative questions, and it could be argued that more rigorous criteria could have been used here. As previously mentioned, the data were obtained from a selective sample population, and the nature of cross-sectional studies precludes inferences of causality. Although 500 psychiatrists were selected for the study and agreed to participate, $101 \mathrm{did}$ not include patients and no information is available concerning the reasons for these recruitment limitations. The survey design may have influenced these missing data and it should be considered as a selection bias, potentially affecting the results. However, this limitation could be counterbalanced by the large sample size that included 1,887 inpatients and outpatients in naturalistic conditions, recruited by 399 psychiatrists in daily practice. With more than 1,000 patients being included in the survey, this is likely to have provided a sample of sufficient size to expect that the results may be generalizable to similar populations.

\section{Conclusion}

Treatment of psychotic disorders aims to obtain functional remission and minimize relapse. Non-adherence to medication is a major risk factor for relapse, and in this study, non-adherence to oral antipsychotic treatment prompted many psychiatrists to switch their patients to long-acting injectable risperidone. Understanding factors associated with poor medication adherence may help physicians to better manage their patients, thereby improving outcomes. Evaluating medication adherence and therapeutic alliance with the MAQ and 4PAS questionnaires, respectively, is straightforward and can provide valuable information in this regard. Given the positive associations between good medication adherence and both therapeutic alliance and 
insight, through the regular monitoring associated with its use, long-acting injectable risperidone can help to improve outcomes by fostering the therapeutic alliance between patients and physicians, particularly in patients with lower insight into their condition. ${ }^{20}$

\section{Acknowledgments}

This study was supported by Janssen-Cilag, France. The authors thank Andy Lockley of Bioscript Medical, UK, who provided editing support for this manuscript, funded by Janssen-Cilag, France. Some data in this manuscript previously appeared in a French language paper. ${ }^{51}$

\section{Disclosure}

FJB and AT have no conflicts of interest in this study. SB is an employee of Janssen-Cilag, France. DM received funding from Janssen-Cilag, France, to develop methodological aspects of this study.

\section{References}

1. Lieberman JA, Perkins D, Belger A, et al. The early stages of schizophrenia: speculations on pathogenesis, pathophysiology, and therapeutic approaches. Biol Psychiatry. 2001;50(11):884-897.

2. Llorca PM. Partial compliance in schizophrenia and the impact on patient outcomes. Psychiatry Res. 2008;161(2):235-247.

3. Robinson DG, Woerner MG, Alvir JMJ, Bilder RM, Hinrichsen GA, Lieberman JA. Predictors of medication discontinuation by patients with first-episode schizophrenia and schizoaffective disorder. Schizophr Res. 2002;57(2-3):209-219.

4. Birchwood M, Todd P, Jackson C. Early intervention in psychosis. The critical period hypothesis. Br J Psychiatry Suppl. 1998;172(33):53-59.

5. Acosta FJ, Bosch E, Sarmiento G, Juanes N, Caballero-Hidalgo A, Mayans T. Evaluation of noncompliance in schizophrenia patients using electronic monitoring (MEMS) and its relationship to sociodemographic, clinical and psychopathological variables. Schizophr Res. 2009;107(2-3):213-217.

6. Bodén R, Brandt L, Kieler H, Andersen M, Reutfors J. Early non-adherence to medication and other risk factors for rehospitalization in schizophrenia and schizoaffective disorder. Schizophr Res. 2011;133(1-3): $36-41$.

7. Misdrahi D, Petit M, Blanc O, Bayle F, Llorca PM. The influence of therapeutic alliance and insight on medication adherence in schizophrenia. Nord J Psychiatry. 2012;66(1):49-54.

8. Velligan DI, Weiden PJ, Sajatovic M, et al. The expert consensus guideline series: adherence problems in patients with serious and persistent mental illness. J Clin Psychiatry. 2009; 70 Suppl 4:1-46; quiz 47-48.

9. Novick D, Haro JM, Suarez D, Perez V, Dittmann RW, Haddad PM. Predictors and clinical consequences of non-adherence with antipsychotic medication in the outpatient treatment of schizophrenia. Psychiatry Res. 2010;176(2-3):109-113.

10. Weiden P, Glazer W. Assessment and treatment selection for "revolving door" inpatients with schizophrenia. Psychiatr Q. 1997;68(4): 377-392.

11. Fleischhacker WW, Oehl MA, Hummer M. Factors influencing compliance in schizophrenia patients. J Clin Psychiatry. 2003;64 Suppl 16:10-13.

12. Mohamed S, Rosenheck R, McEvoy J, Swartz M, Stroup S, Lieberman JA. Cross-sectional and longitudinal relationships between insight and attitudes toward medication and clinical outcomes in chronic schizophrenia. Schizophr Bull. 2009;35(2):336-346.
13. Rocca P, Crivelli B, Marino F, Mongini T, Portaleone F, Bogetto F. Correlations of attitudes toward antipsychotic drugs with insight and objective psychopathology in schizophrenia. Compr Psychiatry. 2008;49(2):170-176.

14. Schennach R, Meyer S, Seemüller F, et al. Insight in schizophreniacourse and predictors during the acute treatment phase of patients suffering from a schizophrenia spectrum disorder. Eur Psychiatry. 2012; 27(8):625-633.

15. Day JC, Bentall RP, Roberts C, et al. Attitudes toward antipsychotic medication: the impact of clinical variables and relationships with health professionals. Arch Gen Psychiatry. 2005;62(7):717-724.

16. Lecomte T, Spidel A, Leclerc C, MacEwan GW, Greaves C, Bentall RP. Predictors and profiles of treatment non-adherence and engagement in services problems in early psychosis. Schizophr Res. 2008;102(1-3):295-302.

17. Misdrahi D, Verdoux H, Lançon C, Bayle F. The 4-Point ordinal Alliance Self-report: a self-report questionnaire for assessing therapeutic relationships in routine mental health. Compr Psychiatry. 2009;50(2): 181-185.

18. Weiss KA, Smith TE, Hull JW, Piper AC, Huppert JD. Predictors of risk of nonadherence in outpatients with schizophrenia and other psychotic disorders. Schizophr Bull. 2002;28(2):341-349.

19. Llorca PM, Abbar M, Courtet P, Guillaume S, Lancrenon S, Samalin L. Guidelines for the use and management of long-acting injectable antipsychotics in serious mental illness. BMC Psychiatry. 2013;13:340.

20. Kaplan G, Casoy J, Zummo J. Impact of long-acting injectable antipsychotics on medication adherence and clinical, functional, and economic outcomes of schizophrenia. Patient Prefer Adherence. 2013; 7:1171-1180.

21. Leucht C, Heres S, Kane JM, Kissling W, Davis JM, Leucht S. Oral versus depot antipsychotic drugs for schizophrenia - a critical systematic review and meta-analysis of randomised long-term trials. Schizophr Res. 2011;127(1-3):83-92.

22. Leucht S, Heres S, Kissling W, Davis JM. Evidence-based pharmacotherapy of schizophrenia. Int J Neuropsychopharmacol. 2011;14(2): 269-284.

23. Tiihonen J, Haukka J, Taylor M, Haddad PM, Patel MX, Korhonen P. A nationwide cohort study of oral and depot antipsychotics after first hospitalization for schizophrenia. Am J Psychiatry. 2011;168(6):603-609.

24. Guy W. ECDEU Assessment Manual for PsychopharmacologyRevised. Rockville, Md: USDHEW; 1976.

25. Targum SD, Houser C, Northcutt J, Little JA, Cutler AJ, Walling DP. A structured interview guide for global impressions: increasing reliability and scoring accuracy for CNS trials. Ann Gen Psychiatry. 2013;12(1):2.

26. Kay SR, Fiszbein A, Opler LA. The positive and negative syndrome scale (PANSS) for schizophrenia. Schizophr Bull. 1987;13(2):261-276.

27. Morisky DE, Green LW, Levine DM. Concurrent and predictive validity of a self-reported measure of medication adherence. Med Care. 1986; 24(1):67-74.

28. Kemp R, Hayward P, Applewhaite G, Everitt B, David A. Compliance therapy in psychotic patients: randomised controlled trial. BMJ. 1996;312(7027):345-349.

29. SAS, Institute Inc. SAS/STAT ${ }^{\circledR}$ Software: Changes and Enhancements, Release 8.2. Cary NC SAS Inst Inc.; 2001.

30. Meyer JM. Understanding depot antipsychotics: an illustrated guide to kinetics. CNS Spectr. 2013;18 Suppl 1:58-67; quiz 68.

31. Barbui C, Kikkert M, Mazzi MA, et al. Comparison of patient and clinician perspectives in the assessment of antipsychotic medication adherence. Psychopathology. 2009;42(5):311-317.

32. Hamann J, Lipp ML, Christ-Zapp S, Spellmann I, Kissling W. Psychiatrist and Patient Responses to Suspected Medication Nonadherence in Schizophrenia Spectrum Disorders. Psychiatr Serv. 2014;65(7): 881-887.

33. Kikkert MJ, Schene AH, Koeter MW, et al. Medication adherence in schizophrenia: exploring patients', carers' and professionals' views. Schizophr Bull. 2006;32(4):786-794. 
34. Osterberg L, Blaschke T. Adherence to medication. $N$ Engl J Med. 2005;353(5):487-497.

35. Kampman O, Lehtinen K. Compliance in psychoses. Acta Psychiatr Scand. 1999;100(3):167-175.

36. Thomas P, Alptekin K, Gheorghe M, Mauri M, Olivares JM, Riedel M. Management of patients presenting with acute psychotic episodes of schizophrenia. CNS Drugs. 2009;23(3):193-212.

37. Wittorf A, Jakobi U, Bechdolf A, et al. The influence of baseline symptoms and insight on the therapeutic alliance early in the treatment of schizophrenia. Eur Psychiatry. 2009;24(4):259-267.

38. Rossi G, Frediani S, Rossi R, Rossi A. Long-acting antipsychotic drugs for the treatment of schizophrenia: use in daily practice from naturalistic observations. BMC Psychiatry. 2012;12:122.

39. Alhewiti A. Adherence to Long-Term Therapies and Beliefs about Medications. Int J Family Med. 2014;2014:479596.

40. Gatti ME, Jacobson KL, Gazmararian JA, Schmotzer B, Kripalani S. Relationships between beliefs about medications and adherence. Am J Health Syst Pharm. 2009;66(7):657-664.

41. Csernansky JG, Schuchart EK. Relapse and rehospitalisation rates in patients with schizophrenia: effects of second generation antipsychotics. CNS Drugs. 2002;16(7):473-484.

42. De Hert M, van Winkel R, Wampers M, Kane J, van Os J, Peuskens J. Remission criteria for schizophrenia: evaluation in a large naturalistic cohort. Schizophr Res. 2007;92(1-3):68-73.

43. Kampman O, Laippala P, Väänänen J, et al. Indicators of medication compliance in first-episode psychosis. Psychiatry Res. 2002;110(1): $39-48$.

44. Mintz AR, Dobson KS, Romney DM. Insight in schizophrenia: a metaanalysis. Schizophr Res. 2003;61(1):75-88.
45. Mutsatsa SH, Joyce EM, Hutton SB, Barnes TR. Relationship between insight, cognitive function, social function and symptomatology in schizophrenia: the West London first episode study. Eur Arch Psychiatry Clin Neurosci. 2006;256(6):356-363.

46. Segarra R, Ojeda N, Peña J, et al. Longitudinal changes of insight in first episode psychosis and its relation to clinical symptoms, treatment adherence and global functioning: one-year follow-up from the Eiffel study. Eur Psychiatry. 2012;27(1):43-49.

47. Charpentier A, Goudemand M, Thomas P. L'alliance thérapeutique, un enjeu dans la schizophrénie [Therapeutic alliance, a stake in schizophrenia]. L'Encéphale. 2008;35(1):80-89. French.

48. Schennach-Wolff R, Jäger M, Seemüller F, et al. Attitude towards adherence in patients with schizophrenia at discharge. J Psychiatr Res. 2009;43(16):1294-1301.

49. Staring AB, Mulder CL, Duivenvoorden HJ, De Haan L, Van der Gaag M. Fewer symptoms vs more side-effects in schizophrenia? Opposing pathways between antipsychotic medication compliance and quality of life. Schizophr Res. 2009;113(1):27-33.

50. Yang J, Ko YH, Paik JW, et al. Symptom severity and attitudes toward medication: impacts on adherence in outpatients with schizophrenia. Schizophr Res. 2012;134(2-3):226-231.

51. Misdrahi D, Delgado A, Bouju S, Comet D, Chiariny JF. Motifs et délais pour l'initiation de la forme injectable à libération prolongée de la rispéridone : enquête auprès de 399 psychiatres français [Rationale for the use of long-acting injectable risperidone: a survey of French psychiatrists]. Encephale. 2013;39 Suppl 1:S8-S14. French.
Patient Preference and Adherence

\section{Publish your work in this journal}

Patient Preference and Adherence is an international, peer-reviewed, open access journal that focuses on the growing importance of patient preference and adherence throughout the therapeutic continuum. Patient satisfaction, acceptability, quality of life, compliance, persistence and their role in developing new therapeutic modalities and compounds to optimize

\section{Dovepress}

clinical outcomes for existing disease states are major areas of interest for the journal. This journal has been accepted for indexing on PubMed Central. The manuscript management system is completely online and includes a very quick and fair peer-review system, which is all easy to use. Visit http://www. dovepress.com/testimonials.php to read real quotes from published authors. 